\title{
A Rare Presentation of Malignant Hyperthermia in a Patient With Poliomyelitis
}

\author{
Sameera Syed ${ }^{\mathrm{a}, \mathrm{b}}$, Mohammad Ansaria ${ }^{\mathrm{a}}$, Bharadwaj Nivarthi ${ }^{\mathrm{a}}$, Nikunj Patel ${ }^{\mathrm{a}}$, Kanwal Farooq ${ }^{\mathrm{a}}$, \\ Jaspreet Kaura, Bushra Komala ${ }^{\mathrm{a}}$, Wageeh Azer ${ }^{\mathrm{a}}$, Shuvendu Sen ${ }^{\mathrm{a}}$
}

\begin{abstract}
Malignant hyperthermia $(\mathrm{MH})$ is a rare hypermetabolic response to halogenated anesthetic gases or succinylcholine. We presented a case of malignant hyperthermia in a 39-year-old male with a past medical history significant for poliomyelitis, who had been exposed to anesthetic agents multiple times in the past. The patient presented with $\mathrm{MH}$ crisis about $4 \mathrm{~h}$ after surgery, with pre operative exposure to succinylcholine. Seventy percent of MH cases are familial ones and sporadic cases are rare. This case report creates awareness of the diagnosis of $\mathrm{MH}$ in patients without family history of the disorder and with delayed symptom onset. Studies are lacking on the pathophysiology of $\mathrm{MH}$ in patients with neuromuscular disorders since these patients might have atypical presentation.
\end{abstract}

Keywords: Malignant hyperthermia; Poliomyelitis; Succinylcholine; Dantrolene

\section{Introduction}

Malignant hyperthermia (MH) is defined as a disturbance of the skeletal muscle calcium homeostasis, triggered by volatile anesthetics and depolarizing muscle relaxants (i.e., all halogenated inhalational anesthetics such as halothane, isoflurane, sevoflurane and the depolarizing muscle relaxant succinylcholine) .The gene for the ryanodine receptor $R Y R 1$ is the primary site for mutations linked with $\mathrm{MH}$, but other genetic loci have been identified such as CACNA1S and STAC3 [1]. Clinical features of MH include: tachycardia, tachypnea, hypoxemia, hypercarbia, metabolic and respiratory acidosis, hyperkalemia, cardiac dysrhythmias, hypotension, skeletal muscle rigidity, and hyperthermia. The main stay of treatment for MH is im-

Manuscript submitted April 11, 2018, accepted June 12, 2018

aDepartment of Internal Medicine, Raritan Bay Medical Center, Perth Amboy, NJ 08861, USA

${ }^{\mathrm{b}}$ Corresponding Author: Sameera Syed, Department of Internal Medicine, Raritan Bay Medical Center, 530 New Brunswick Avenue, Perth Amboy, NJ 08861, USA. Email: sameera.syed@hackensackmeridian.org

doi: https://doi.org/10.14740/jmc3083w mediate dantrolene administration [1] and other supportive measures in correcting hyperthermia, acidosis, hypoxemia, arrhythmias and preserving renal function. Supportive measures include: providing a patent airway by intubation, cooling blanket for hyperthermia, anti-hypertensive medication drips for hypertension, adequate IV fluid hydration etc.

\section{Case Report}

A 39 years old male with a past medical history significant for poliomyelitis of the left lower extremity was admitted for elective perianal fistulotomy for an unhealing perianal fistula. Due to poliomyelitis, the patient had left lower extremity atrophy and weakness. Patient had six minor knee surgeries and appendectomy without any issues. During the procedure, the patient was intubated and received fentanyl, lidocaine, succinylcholine, propofol. Patient had an uncomplicated extubation in the recovery room. Patient was recovering well with normal vital signs until $3 \mathrm{~h}$ postoperative. He complained of shaking, chills and gradually his temperature went up to $40.1{ }^{\circ} \mathrm{C}$ in the subsequent $2 \mathrm{~h}$. Four hours postoperative, his vitals were: blood pressure (BP) 200/110 mm Hg, pulse $125 \mathrm{bpm}$, RR 33 breaths $/$ min, $\mathrm{T} 40^{\circ} \mathrm{C}$. The patient was immediately transferred to ICU. Patients labs revealed potassium: 5.1; anion gap: 15; CK: 2089, arterial pH: 7.15; arterial $\mathrm{pCO}_{2}: 58$; arterial $\mathrm{pO}_{2}: 67$; arterial $\mathrm{pHCO}_{3}: 19.8 ; \mathrm{FiO}_{2}: 55 \% ; \mathrm{SpO}_{2}: 86 \%$.

Based on the clinical and lab findings a diagnosis of $\mathrm{MH}$ was established. Patient was immediately intubated and received IV dantrolene $2.5 \mathrm{mg} / \mathrm{kg}$; cold normal saline infusion, cooling blankets and was started on labetalol drip. Patient was off labetalol drip in $2 \mathrm{~h}$ but his temperature fluctuated between $38.3-39.2{ }^{\circ} \mathrm{C}$ over the next 2 days. Patient's blood cultures showed no growth. TSH and cortisol levels were within normal limits. Over the next 8 days patient's hospital course was complicated by electrolyte imbalances, respiratory acidosis requiring prolonged intubation, DIC, rhabdomyolysis, fluid overload and acute renal failure. After prolonged ventilator and supportive management the patient was completely weaned off all sedatives/hypnotics and passed the spontaneous breathing trial. On the eighth day of the hospital stay the patient got extubated and was discharged on 2 days later. There was a gradual decline in hemoglobin from 13 to 7.9 and platelet count from 253 to 66 over the next 6 days of dantrolene infusion, which is the most common complication of dantrolene. 
The patient's blood dyscracia slowly improved and reached baseline in 1 month.

\section{Discussion}

The incidence of $\mathrm{MH}$ episodes during anesthesia is between $1: 10,000$ and $1: 250,000$ [1]. The syndrome is inherited in an autosomal dominant pattern, although wide variability is reported [1]. The incidence of $\mathrm{MH}$ is 1 in 30,000 estimated by Robinson et al [2]. An uncontrolled rise of myoplasmic calcium, which activates biochemical processes related to muscle activation is responsible for the pathophysiologic changes in $\mathrm{MH}$. A defective $\mathrm{Ca} 2+$ channel located in the SR membrane is implicated in MH susceptibility. This channel is termed as ryanodine receptor (RyR1). In most cases, the syndrome is caused by a defect in the ryanodine receptor. Over 400 variants have been identified in the RYR1 gene located on chromosome 19q13.1, and at least 34 are causal for $\mathrm{MH}$ [1]. The channel RyR1 is closely associated with many other proteins, such as the dihydropyridine receptor (DHPR) $\mathrm{Ca} 2+$ channel which is situated in the T-tubule region of the sarcolemma that mediates transfer of voltage change to the RyR1 receptor. Other proteins with potential or known roles in RyR1 function include integral SR membrane proteins i.e., SRP-27, junctate, the transient receptor potential cation channel (TRPC) family and triadin [1]. Proteins that modulate the function of RyR1 include the FK508 binding protein FKBP12 , the $\mathrm{Ca} 2+$ binding protein calmodulin, the histidine-rich $\mathrm{Ca} 2+$ protein, $\mathrm{HRC}$ and the luminal $\mathrm{Ca} 2+$ buffer calsequestrin. $\mathrm{HRC}$ is also a luminal protein known to interact with both triadin and SERCA and has been suggested to have a role in mediating cross talk between SR Ca2+ uptake and release [1].

Few myopathies are associated with increased susceptibility to $\mathrm{MH}$ with confirmed $R Y R 1$ variants. These myopathies include: central core disease (CCD), multiminicore myopathy $(\mathrm{MmD})$ and centronuclear myopathy [1]. Recessive variants in $R Y R 1$ have been associated with $\mathrm{MmD}$, some of which result in altered $\mathrm{Ca} 2+$ release from intracellular stores. Similarly there might be few variants of $R Y R 1$ in neuromuscular disorders like poliomyelitis which may enhance susceptibility or may be associated with delayed onset. However studies are lacking.

About 528 cases of $\mathrm{MH}$ were analyzed by North American $\mathrm{MH}$ registry out of which 64 cases of $\mathrm{MH}$ were postoperative and the mean time for symptom onset was $0-40 \mathrm{~min}$ for those cases [3]. MH may occur at any time during anesthesia as well as in the early postoperative period, but usually not $1 \mathrm{~h}$ after discontinuation of volatile agents [3]. However in our case, the patient developed $\mathrm{MH}$ after $4 \mathrm{~h}$ of succinylcholine administration, a unique presentation for $\mathrm{MH}$.

An increase in the end-tidal carbon dioxide is a sensitive and early sign of MH [4]. Uncontrolled hypermetabolism leads to respiratory and in most cases metabolic acidosis due to rapid consumption of energy stores and ATP. MH if untreated leads to rhabdomyolysis, life-threatening hyperkalemia; myoglobinuria, and acute renal failure. Additional life-threatening complications include DIC, congestive heart failure, bowel ischemia, and compartment syndrome of the limbs secondary to profound muscle swelling.

The "gold standard" for diagnosis of $\mathrm{MH}$ is currently an in vitro contracture test (IVCT), which is based on contracture of muscle fibers in the presence of halothane or caffeine. An individual is considered susceptible to $\mathrm{MH}$ when both caffeine and halothane test results are positive. DNA analysis, however, offers an alternative to the IVCT, requiring only a blood specimen.

Dantrolene is the only drug known to specifically treat MH. Dantrolene inhibits the DHPR in an RyR1-dependent manner, binding to a specific site on the RyR1 protein and reducing RyR1 channel activity in intact muscle cells $[5,6]$. Hyperthermia is treated with cooling blankets and cold normal saline infusion; and arrhythmias are treated with amiodarone. Electrolyte abnormalities need to be addressed, ventiltory support may be needed and other supportive measures also come into play [1].

\section{Conclusions}

In patients with neuromuscular disorders like poliomyelitis, succinylcholine should be avoided and caution should be exercised with administration of inhalational agent to avoid $\mathrm{MH}$. The susceptibility and timeline between exposure to the triggering agent and symptom onset of $\mathrm{MH}$ in patients with neuromuscular disorders may be related to $R Y R 1$ variants similar to muscular dystrophies. However studies are lacking. $\mathrm{MH}$ should be high on the differential in cases with the appropriate signs and symptoms suggestive of $\mathrm{MH}$, after there is recurrent exposure to anesthetic agents, no matter what the time line is.

\section{References}

1. Rosenberg H, Pollock N, Schiemann A, Bulger T, Stowell K. Malignant hyperthermia: a review. Orphanet J Rare Dis. 2015;10:93.

2. Robinson RL, Curran JL, Ellis FR, Halsall PJ, Hall WJ, Hopkins PM, Iles DE, et al. Multiple interacting gene products may influence susceptibility to malignant hyperthermia. Ann Hum Genet. 2000;64(Pt 4):307-320.

3. Litman RS, Flood CD, Kaplan RF, Kim YL, Tobin JR. Postoperative malignant hyperthermia: an analysis of cases from the North American Malignant Hyperthermia Registry. Anesthesiology. 2008;109(5):825-829.

4. Pollock AN, Langton EE, Couchman K, Stowell KM, Waddington M. Suspected malignant hyperthermia reactions in New Zealand. Anaesth Intensive Care. 2002;30(4):453-461.

5. Bannister RA. Dantrolene-induced inhibition of skeletal L-type Ca2+ current requires RyR1 expression. Biomed Res Int. 2013;2013:390493.

6. Paul-Pletzer K, Yamamoto T, Bhat MB, Ma J, Ikemoto N, Jimenez LS, Morimoto H, et al. Identification of a dantrolene-binding sequence on the skeletal muscle ryanodine receptor. J Biol Chem. 2002;277(38):34918-34923. 